\title{
Current Review of Delta Variant of SARS-CoV-2
}

\author{
Selia Chowdhury, Mehedi Hasan Bappy, Samia Chowdhury, Md. Shahraj Chowdhury, and Nurjahan \\ Shipa Chowdhury
}

\section{ABSTRACT}

Mutations are causing SARS-CoV-2 to alter its genetic structure to improve its potential to elude the immune system, making vaccine buildout against the virus more difficult. Multiple SARS-CoV-2 variants have been found up to this point; based on their impact on public health some are considered variants of concern (VOCs) and some are considered variants of interests. VOCs are linked to superior transmissibility, a decline in neutralization by natural or vaccine induced antibodies, evading capability of detection, and a reduction in the efficacy of vaccines or therapeutics. In this article, a SARS-CoV-2 subtype, known as Delta, has been revised to provide the current state of the art and an appropriate foundation for future research works. The evolution, pathogenesis, current trends of transmission, associated symptoms, suggested prevention and treatments, and vaccine efficacy of Delta variant are reviewed and discussed.

Keywords: COVID-19, delta, variants, treatment, vaccine efficacy.

Submitted : October 24, 2021

Published. : November 23, 2021

ISSN: 2593-8339

DOI: $10.24018 /$ ejmed.2021.3.6.1120

S. Chowdhury*

Dhaka Medical College, Dhaka, Bangladesh.

(e-mail: chowdhury.selia@gmail.com)

M. H. Bappy

University of Iowa, Iowa City, IA, USA.

S. Chowdhury

Sylhet MAG Osmani Medical College, Sylhet, Bangladesh.

M. S. Chowdhury

DSHE, Education Ministry, Sylhet, Bangladesh.

N. S. Chowdhury

Dinajpur Medical College, Dinajpur, Bangladesh.

*Corresponding Author

\section{INTRODUCTION}

COVID-19 has been a nightmare to billions of people all over the world impacting their lives with unprecedented losses be it economic, physical, or mental. As of October 20, 2021, there have been about 244 million cases and 4.95 million deaths due to COVID; United States, India, Brazil, United Kingdom, and Russia are leading the list for most COVID cases [1]. New cases on October 21, 2021, show that UK, Russia, Turkey, and Romania are following USA, Fig. 1 shows a comparison of total and new cases categorized by countries. Although, there have been vaccines that are very effective to protect against coronavirus, the number of cases and deaths are still rising. One critical reason behind this increase is the mutation capability of the virus. SARS-CoV-2 has been going through several genomic alterations and is strongly spreading all over the world despite the prevention and vaccination efforts. A total of twelve variants of SARSCoV-2 have been documented until now, they are Alpha, Beta, Gamma, Delta, Delta Plus, Epsilon, Eta, Theta, Iota, Kappa, Lambda, and Mu. World Health Organization (WHO) is categorizing these variants depending on their transmissibility, virulence, and overall impact on health to prioritize worldwide surveillance and investigation, and eventually to report on the enduring retort to the deadly disease COVID-19 [2].

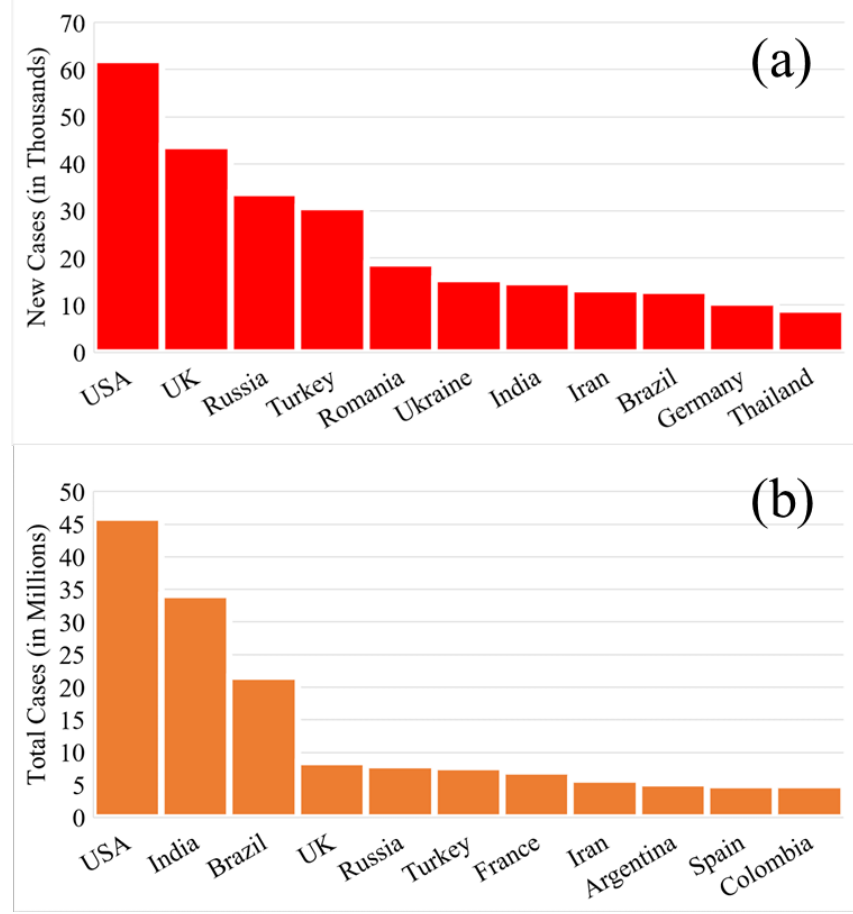

Fig. 1. (a) Total and (b) new COVID-19 cases reported so far, categorized by countries.

A variant of interest (VOI) is a SARS-CoV-2 virus with genomic variations affecting viral properties such as communicability, the severity of disease, immunological evasion, diagnostic or therapeutic avoidance; and that have 
been found to trigger substantial community transmission or multiple clusters of COVID-19, in several countries with a gradually growing comparative incidence coupled with swelling numbers of cases or other obvious epidemiological implications that point to an evolving risk to worldwide public health [2]. Presently, Eta, Iota, Kappa, Lambda, Mu, and all their descendent lineages are designated as the variants of interest. A variant of concern (VOC) is a SARSCoV-2 subtype which satisfies the definition of VOI and additionally causes an increase in transmission ability or an unfavorable change in the epidemiology of COVID-19 or an increase in virulence or a modification in the clinical picture of the disease or a reduction in the effectiveness of social and public health measures or diagnoses, vaccines, and therapeutics available [2]. Alpha, Beta, Gamma, Delta, and all their descendent subvariants are currently labeled as the VOC.

The Delta variant is the most highly contagious variant of COVID-19 and is currently the predominant variant all around the world which makes it the most significant one to focus on. Delta variant is twice more infectious than other variants, more resistant to health preventions such as isolation, causing more wide-ranging and severe symptoms amid patients. Patients who are contaminated with the variant Delta are prone to be hospitalized than patients infected with other variants. The highest risk of transmission remains in unvaccinated people, who become infected much more frequently and consequently spread the virus. [3]. Delta variant is also causing breakthrough infections in the vaccinated people, but it is less frequent than infections in unvaccinated people. Another concerning issue is that people infected with the Delta variant, whether they are vaccinated or not, can transmit the virus to others. It is also spreading more easily among children [4]. The origin of this variant is India; it was first documented in October 2020. WHO declared Delta as a variant of interest on April 4, 2021, then it was declared as a variant of concern on May 11, 2021 [2]. As of October 21, 2021, United States has submitted the highest number of Delta Variant sequences to GISAID, the number being 642,725. United Kingdom has submitted 624,244 cases while Germany has 82,453 and Denmark has 59,196 sequences submitted [5]. According to the Centers for Disease Control and Prevention (CDC), Delta represented barely $0.1 \%$ of cases in the USA by early April. Delta was responsible for $1.3 \%$ of cases by early May and this number had risen to $9.5 \%$ by early June. According to GISAID, approximately $98.5 \%$ of the genomic sequence from USA correspond to Delta variant. In contrast, UK, Germany, Denmark, Sweden, Turkey, and Japan have almost 100\% of their cases identified as Delta. By September 21, 2021, Delta had dispersed to 185 countries and became the globally most prevalent variant [6]. It can be surmised that Delta variant is the most dangerous among all the SARS-Cov-2 viruses with increased transmission rate and muted virulence. Reasonably, there has been significant number of works on the Delta variant, the state of the art of structure and mutation of this variant is presented within this review for the convenience of the scientific community.

\section{The Delta Variant: Structure And Mutations}

Adaptive changes in the genome can modify the pathogenic potential of a virus. When an animal or person is infected, mutations in the genetic coding of the virus develop spontaneously over time. It's critical to keep an eye on circulating viruses for significant mutations in key areas of the genome [7], [8]. Even a little alteration in amino acid sequence can significantly alter the capacity of a virus to elude the immune system and hinder the advancement of vaccine development. Similar to all other RNA viruses, SARS-CoV-2 is vulnerable to genetic progression during its adaptations to novel hosts. As a result, numerous variants with different characteristics than the inherited strains may arise. The Delta configuration has developed stronger than the initial strains, allowing it to spread quicker, induce more intense symptoms of COVID-19-related illness, cause breakthrough infection, and have a higher mortality rate [9]. Many mutations, on the other hand, have little effect on the virus's capacity to propagate or cause disease because they do not change the main proteins involved in infection; eventually, these variations are outcompeted by variants with more advantageous changes [10].

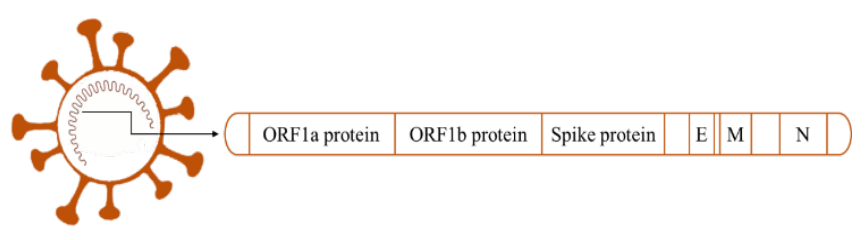

Fig. 2. A SARS-CoV-2 genome map.

In Fig. 2, the genome of the virus is enclosed in an envelope containing proteins, including the spike protein. Different sections represent genes in the genome of SARSCoV-2 (right) that carry instructions for constructing viral components. According to a sequence study of its isolates, the genome can encrypt up to fourteen ORFs (open-reading frames). ORF1a and ORF1ab encode polyproteins that autoproteolytically disintegrate into sixteen non-structural proteins which form the replicase-transcriptase complex [11]. At the end of the viral genome, up to thirteen ORFs are expressed from nine putative sub-genomic RNAs [11]. Spike $(\mathrm{S})$, envelope $(\mathrm{E})$, membrane $(\mathrm{M})$, and nucleocapsid $(\mathrm{N})$ are four structural proteins, and nine potential accessory factors are located among them. The spike protein section contains genetic instructions for constructing the spike protein granting SARS-CoV-2 to adhere to human cells during infection. This portion of the genome is crucial for tracking mutations [8].

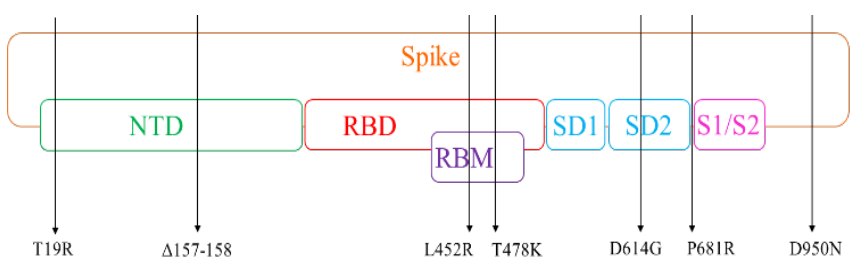

Fig. 3. SARS-CoV-2 Delta variant amino acid mutations displayed on a SARS-CoV-2 genome map with an emphasis on Spike. NTD: N-Terminal Domain, RBD: Receptor Binding Domain, RBM: Receptor-Binding Motif, SD1 and SD2: Subdomains 1 and 2, S1/S2: Protease Cleavage Site 1 and 2.

The variant Delta is alternatively recognized as B.1.617.2, 
comprises constructively selected mutations in the spike protein, the mutations include T478K, P681R, L452R, as well as the D614G. These mutations may be regarded Delta variant hallmark characteristics [12]. Fig. 3 depicts amino acid mutations of Delta variant displayed on a SARS-CoV-2 genome map focusing on Spike. Spike protein is the biggest of coronavirus's four main structural proteins. The spike protein forms trimers, which then form large structures termed spikes or peplomers that protrude from the virion's surface [9]. The virus family gets its name from the unique look of these spikes when seen using negative stain transmission electron microscopy resembles the solar corona. These spikes must locate receptors in human cells to attach to; investigations have revealed that these spikes bind to ACE-2 receptors. The infection spreads by reproducing the virus's genetic code once these spike proteins have unlocked the cells. There are 13 mutations in the B.1.617.2 genome that cause changes in the amino acid sequences of the encoded proteins [9]. The spike protein substitutions corresponding to the Delta variant are T478K, T19R, P681R, D614G, T95I, D950N, G142D, R158G, L452R, F157-, E156-, (A222V*), (V70F*), (K417N*), and (W258L*), according to CDC. Particularly concerning are four of them inside the spike protein code of the virus [9].

1. L452R: The leucine-to-arginine change at position 452 gives higher attraction of the spike protein for the ACE2 receptor as well as reduced immune system identification capacity. These changes are not unique to the variation when considered separately; rather, their simultaneous presence is.

2. T478K: A threonine-to-lysine substitution occurs at position 478.

3. D614G: The aspartic acid-to-glycine alteration at position 614 is communal to other highly transmissible variants such as Alpha, Beta, and Gamma.

4. P681R: The proline-to-arginine substitution at position 681 may increase the variant's cell-level infectivity by enabling cleavage of the $\mathrm{S}$ precursor protein to the working S1/S2 configuration.

Major changes in the Delta variant discussed above make it simpler for the virus's spikes to bind to ACE-2 receptors. This means it can infect and multiply more quickly, as well as more effectively escape the body's natural disease-fighting defenses. According to the WHO, the spike protein changes make the Delta variation the fittest version yet. Furthermore, the presence of two medically significant point mutations, P681R and D950N, in these LCRs, along with their remarkable conservation, implies that they might be a characteristic linked to the fast spread of the highly infectious Delta lineage [13].

The Delta variant has gone through several major mutations in the spike protein and spread into a few subtypes, which are being classified as Delta Plus. AY.1, AY.2, and AY.3 are referred to as Delta Plus and they are structurally very alike the primary Delta variant, but it contains a few changes [14]. Delta along with Delta Plus variants exhibit distinct mutation patterns; and not just a K417N addition to the Delta makes the Delta Plus [15]. Kannan et al. [15] discovered that the Delta Plus variant possesses a higher percentage of high-prevalence mutations than the Delta variant. Delta Plus has a greater proportion of three hallmark mutations in spike protein: A222V, G142D, and T95I than the Delta. The Delta Plus variation has three additional mutations in the spike protein: W258L, V70F, and K417N. They further recognized a novel mutation in ORF1a (A1146T), exclusively present with 58\% prevalence in the Delta Plus variant. They also reported that five critical mutations are substantially more common in Delta Plus than in Delta: A222V, T95I, G142D, K417N, and R158G.

\section{Clinical Manifestations, Management, AND TREATMENTS}

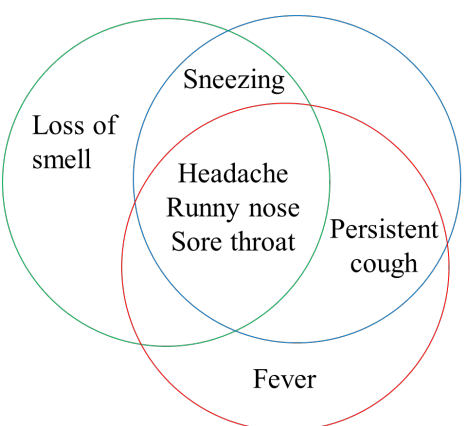

Fig. 4. Top five symptoms of COVID-19 in fully vaccinated (green), partially vaccinated (blue), and unvaccinated (red) population.

There is not much difference in the clinical manifestations of people infected with the delta variant compared to the original COVID strain. Some reports are suggesting that the symptoms are slightly different in the case of Delta variant infection. The most common symptoms of COVID-19 were shortness of breath, cough, fever, change in sense of smell and taste, headache, nausea, vomiting, sore throat, fatigue, muscle/joint aches, and other lung complications [16]. However, cough, loss of smell, nausea, vomiting, and diarrhea are less common with the delta variant and are still being reported in reduced quantities. For Delta variant, the most common symptoms include sore throat, runny nose, sneezing, headache, fever, and persistent cough. A UK based COVID symptoms tracker app 'Zoe's COVID Symptom Study' collected millions of symptom samples from fully vaccinated, single-dose vaccinated, and unvaccinated population [17]. For fully vaccinated people, the topmost common symptoms are headache, runny nose, sneezing, sore throat, and loss of smell. Single dose vaccinated people reported headache, runny nose, sore throat, sneezing, and persistent cough to be the most frequent manifestation of the illness. While unvaccinated people's top symptoms were headache, sore throat, runny nose, fever, and persistent cough. Therefore, headache, sore throat, and runny nose are the most reported symptoms; persistent cough is frequent in case of no/partial vaccination, fever is mostly frequent for unvaccinated people, and sneezing happens for fully/partial vaccinated individuals with COVID-19. Fig. 4 shows top five symptoms of COVID-19 in fully vaccinated, partially vaccinated, and unvaccinated population.

There is currently no specific remedy for COVID-19 and the treatment goal is to alleviate symptoms in mild to moderate cases. Treatment is identical for Delta variant as any other emerging variants, and treatment should be performed based on the individual outcomes of the infected 
patient. Potential treatment can be devised into four categories which needs to be administered at different juncture of the disease course, see Fig. 5.

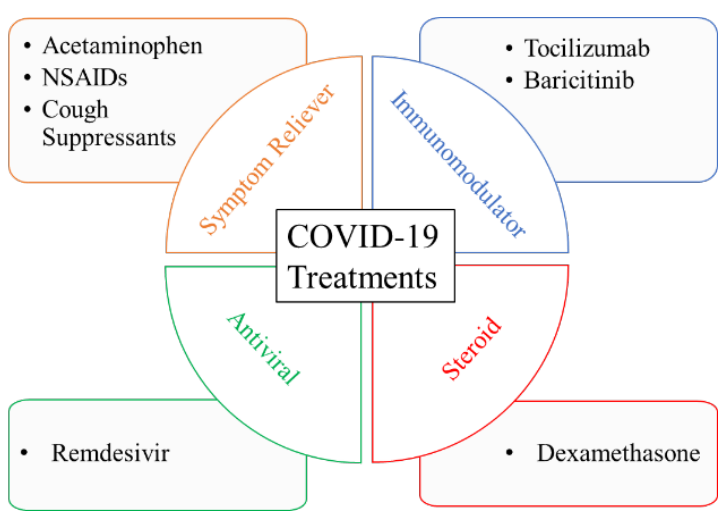

Fig. 5. Four categories of COVID-19 treatment with example medications.

For mild to moderate symptoms, the treatment plan should include category 1: getting appropriate rest, staying hydrated, cough suppressants, and over-the-counter medications such as acetaminophen for relieving fever, aches, and pain. The US Food and Drug Administration (FDA) has authorized treatments for people who have been hospitalized with COVID-19 and who are at risk for developing severe illness [10]. Three monoclonal antibody treatments have emergency use authorization (EUA) by FDA, they are:

1. REGEN-COV: A combination of Casirivimab and Imdevimab [18].

2. A combination of Bamlanivimab and Etesevimab [19], and

3. Sotrovimab [20].

All three treatments have been approved for the treatment of mild to moderate COVID-19 in adults and children aged 12 and up who weigh $40 \mathrm{~kg}$ or more and have positive results from direct SARS-CoV-2 viral testing and are at high risk of developing severe COVID-19, which could result in hospitalization or death [18]-[20]. Note that, these are not approved for use in hospitalized patients due to COVID-19, or patients requiring oxygen treatment due to COVID-19, or patients who require an increase in baseline oxygen flow rate owing to COVID-19 in those on chronic oxygen therapy due to non-COVID-19 related comorbidities [18]-[20]. REGENCOV and Bamlanivimab + Etesevimab cocktail should only be used for post-exposure prophylaxis of COVID-19 [18], [19]. These therapies ought to be given intravenously rapidly after developing symptoms and they can reduce the risk of hospitalization and emergency room visits. People who are unable to produce their own antibodies against the SARSCoV-2 virus should go through monoclonal antibody treatment which can reduce the mortality risk by $20 \%$ [16].

Because of its unique mutation, therapeutics like monoclonal antibody treatments might not be as effective on the Delta and its subvariant cases as the earlier variants. The sensitivity of Delta strain to monoclonal antibodies and to antibodies present in sera from individuals who had recovered from COVID-19 or who had received a COVID19 vaccine have been examined and then compared this strain with other strains of SARS-CoV-2 by [21]. The Delta variant indicated neutralization resistance by some anti-NTD and anti-RBD monoclonal antibodies such as Bamlanivimab while these antibodies revealed reduced adhesion to the spike protein. The authors' claim is that an escape from non-RBD and RBD epitopes of the spike protein targeting antibodies is affecting the rapid spread of the Delta variant. Convalescent plasma from recovered individuals can also have clinical benefits for COVID-19 patients, EUA has been granted for the use of high-titer convalescent plasma among hospitalized patients with COVID-19 who are early in the course of the disease or have impaired humoral immunity [22]. However, as the virus' strain changes, this becomes less effective.

Remdesivir is the only FDA approved antiviral drug that slows down the virus' progression and it is proven beneficial for the most acute patients of COVID-19. It is used on hospitalized patients only and it can only be intravenously administered [23].

Dexamethasone is a steroid that proved to be very useful in severe COVID-19 patients. It is only recommended for severely ill COVID-19 patients with supplemental oxygen or ventilatory support. Data on the efficacy of Dexamethasone and other glucocorticoids showed about 17\% relative reduction in mortality for patients needing oxygen and ventilatory support but showed no conclusive benefit who did not require oxygen or ventilatory support [23].

Baricitinib, a Janus kinase (JAK) inhibitor, is commonly used in treating rheumatoid arthritis. It is believed to have possible antiviral effects besides immunomodulatory effects due to interference with viral entry. Data suggest that it might offer benefits against mortality for patients with severe disease. It is recommended that baricitinib should be an option for high-flow oxygen or noninvasive ventilation requiring patients and for select patients on low-flow oxygen who are advancing toward requiring higher levels of respiratory support instead of dexamethasone initiation [23]. Tofacitinib may also have similar clinical benefits, limited data suggest a lower mortality rate if it is used [23].

For treatment of COVID-19, numerous agents that target the IL-6 pathway such as the IL-6 receptor blockers tocilizumab and sarilumab and the direct IL-6 inhibitor siltuximab have been assessed. Markedly elevated inflammatory markers (Ferritin, D-dimer) and elevated proinflammatory cytokines (interleukin [IL]-6) are related to serious and fatal COVID-19 [23]. By blocking the inflammatory pathway these agents can prevent disease progression [24]. Tocilizumab is suggested as an option for individuals requiring high-flow oxygen or more intensive respiratory support, specifically, patients who have progressively greater oxygen requirements for only COVID19 related reasons. It is intravenously given and showed clear benefits for fatality reduction.

There are other medications with known or believed antiviral or immunomodulating effects that have been proposed for use in COVID-19 patients. Ivermectin, Hydroxychloroquine, Favipiravir, Interferons, IL-1 inhibitors, Azithromycin, Lopinavir-ritonavir, etc. are some examples of them. However, there is insufficient evidence of the clinical benefit of using these drugs, and they are not recommended to be used in COVID-19 patients without any robust clinical evidence [23].

\section{VACCINE EFFICACY}

There have been several studies focused on the 
effectiveness of different vaccines against Delta variant, a clear picture is drawn from these investigations. Current vaccines function against the original Delta variant, but show less effectiveness, especially in older age groups, since they had a slower immune response, and their protection may deteriorate faster. Bernal et al. [25] studied the efficiency of the vaccines BNT162b2 (Pfizer) and ChAdOx1 nCoV19(Oxford-AstraZeneca) against Delta variant. To estimate the efficacy of immunization against the delta variant/the predominant strain (B.1.1.7) induced symptomatic disease, they used test-negative case-control design. After single dose of vaccine (BNT162b2/ChAdOx1 nCoV-19), efficacy was markedly lower amongst delta infected people (30.7\%) compared to the alpha infected people (48.7\%). Both vaccines resulted in analogous outcomes. The full dose effectiveness of the BNT162b2 was $93.7 \%$ for alpha infected people while it was $88.0 \%$ for delta infected people. The twodose efficacy of the ChAdOx $1 \mathrm{nCoV}-19$ was $74.5 \%$ for alpha infected individuals and $67.0 \%$ for delta infected individuals. Comparing delta against alpha, the authors concluded moderate variations in the efficacy of the vaccines after two doses. The absolute differences in the effectiveness of the vaccine were more pronounced after receiving the first dose.

In a report by Moline et al. [26] three currently authorized vaccines in the United States (Pfizer, Moderna, and Johnson and Johnson (J\&J)) showed high effectiveness in preventing laboratory-confirmed COVID-19 associated hospitalizations. For adults 65 years or older, full vaccination efficacy with mRNA vaccines (Pfizer and Moderna) was greater than 91\% while with J\&J it was greater than $84 \%$ [26], [27]. For identification and neutralization of the four variants Alpha, Beta, Gamma, and Delta, Van Gills et al. [28] performed a direct difference assessment of the sera ability from vaccinated people with any one of four vaccines: mRNA1273 (Moderna), BNT162b2 (Pfizer), Ad26.COV2.S (J\&J), or AZD1222 (Oxford-AstraZeneca). Four weeks after completion of vaccination, BNT162b2 and mRNA-1273 recipients showed maximum SARS-CoV-2 wild-type neutralizing antibody titers; it was considerably lesser in the recipients of the adenovirus vector-based vaccines AZD1222 and Ad26.COV2.S. For all age groups, the fold reduction in neutralization of the abovementioned variants in comparison with the wild-type was similar, the largest neutralization capacity decline showed by vaccine sera was against the Beta, Delta, Gamma, and Alpha, following the order. Stowe et al. [29] stated estimates of vaccine efficacy against Delta induced symptomatic disease [25], [29]. After a full vaccination dose, effectiveness went $88 \%$ for the BNT162b2 and $67 \%$ for the ChAdOx1 AZD1222. The efficacy after the first dose was $94 \%$ for BNT162b2 and $71 \%$ for ChAdOx 1 AZD1222, while it increased to $96 \%$ and $92 \%$ for BNT162b2 and ChAdOx1 AZD1222 respectively.

Kumar et al. [30] investigated the clinical outcomes of vaccinated persons among hospitalized patients in India. They examined the clinical attributes and consequences of 1161 hospitalized patients of COVID-19. These patients were vaccinated with COVAXIN (BBV-152) or COVISHIELD (ChAdOx1). The number of vaccinated individuals was 495 and unvaccinated individuals were 666 . More than $90 \%$ of patients in these two groups have infected the Delta variant. Superior neutralizing antibodies and substantially reduced
Ferritin and LDH were found in the vaccinated individuals in comparison with the unvaccinated individuals. Disease severity was $3.2 \%$ for vaccinated group and $7.2 \%$ for unvaccinated group. Also, ventilatory support requirement was lower in the vaccinated group $(2.8 \%)$ compared to the unvaccinated group (5.9\%), although vaccinated individuals were considerably older with other risk factors. Death rate of single dose recipient group $(3.35 \%)$ was similar to the unvaccinated group $(3.45 \%)$, however, death in a fully vaccinated group with breakthrough infections was almost $50 \%$ lower $(1.51 \%)$. They concluded that both vaccines are beneficial to reduce illness severity and death in hospitalized patients who had been fully vaccinated against the Delta variant.

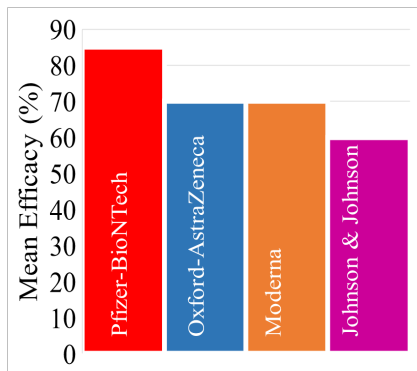

(a) Against symptomatic infection

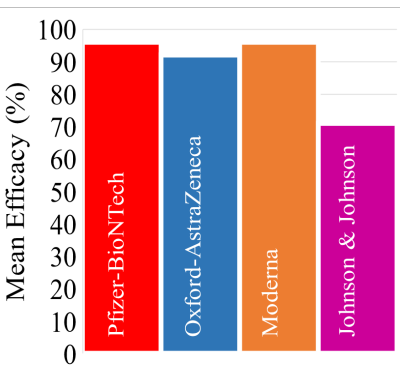

(b) Against severe disease/hospitalization
Fig. 6. Efficacy against delta variant of four COVID-19 vaccines ((a) against symptomatic infection and (b) against severe disease) as reported in [31].

Cevik et al. [31] reported a comparison table of efficacy for different vaccines against Alpha, Beta, Gamma, and Delta variants for symptomatic infection, severe disease, and infection. Against Delta variant, Pfizer-BioNTech provides the most protection, their efficacy being $95 \%$ against severe disease and $85 \%$ against symptomatic infection. Both Moderna and AstraZeneca vaccines have $95 \%$ and $70 \%$ efficacy against severe disease and symptomatic infection respectively. J\&J has $71 \%$ efficacy against severe disease caused by Delta variant and Novavax has 60\% efficacy against symptomatic infection. No efficacy data was reported for Sinovac against Delta variant. Fig. 6 shows these findings in graphical format.

Tregoning et al. [32] discussed some common and rare side effects of vaccination. The common side effects include local/systemic minor acute reactions like swelling, injection pain, fatigue, fever, headaches, and joint and muscle pain. There have also been reports of more serious side effects such as myocarditis, anaphylaxis, and thrombocytopenia [32], [33]. However, the number of severe vaccine-induced complications is very rare, in no way these should obstruct the vaccination of the population. Vaccines are still extremely effective for preventing Delta variant hospitalization and death, according to the CDC [9]. They claim that fully vaccinated persons who have breakthrough infections from Delta variant stay infectious for a shorter length of time, and they advise everyone to be vaccinated and use masks inside in public places to prevent this variant's spread.

\section{CONCLUSION}

The present state of the art and an adequate basis for future research activities have been discussed in this article for a 
variant of SARS-CoV-2 known as Delta. The Delta variant's evolution, etiology, current transmission patterns, related symptoms, proposed management and treatment, and vaccination effectiveness are reviewed and addressed. Through changes in the spike gene, the virus can increase its ability to infect individuals or enable the virus to avoid neutralizing antibodies. Mutations can make the virus weaker or stronger, allowing it to spread quicker or cause more infections. The Delta variation and its descendants can spread SARS-CoV-2 about twice as quickly as the original strain, and the severity of the disease may be higher as well. Clinical manifestations of people infected with the delta variant compared to the other COVID strains remains almost the same. Also, there is no management and treatment specific to Delta variant, treatment options apply irrespective of the variant based on the symptoms and severity of illness. Current studies found the efficacy of existing vaccines against the Delta variant to be lower than the previous variants. However, most of the vaccines work very well to protect the population against severe illness which requires hospitalization. After receiving a COVID-19 vaccine, protection against the virus may wane with time, making it less able to protect against the Delta and other emerging variants. Very recently, authorization for an additional booster dose for fully vaccinated people has been approved, Pfizer-BioNTech was approved on September 22, 2021, and Moderna and J\&J were approved on October 20, 2021 [34], [35]. People who are 65 years or older, or 18 years or older who live in long-term care settings/have underlying medical conditions/work in high-risk settings/live in high-risk settings, are eligible for COVID-19 vaccine booster shots [36]. More studies are being conducted as more data on these variations becomes available, to better understand the polymorphisms and their influence on transmission, hospitalization, vaccination efficacy, and mortality. We hope that adequate study will be done on Delta variant to avoid any negative consequences that they and their mutations may have.

\section{REFERENCES}

[1] Coronavirus Cases [Internet]. Worldometers.info. [cited 2021 Oct 20]. Available from: https://www.worldometers.info/coronavirus/.

[2] Tracking SARS-CoV-2 variants [Internet]. Who.int. [cited 2021 Oct 20]. Available from: https://www.who.int/en/activities/trackingSARS-CoV-2-variants.

[3] CDC. Delta variant: What we know about the science [Internet]. Cdc.gov. 2021 [cited 2021 Oct 21]. Available from: https://www.cdc.gov/coronavirus/2019-ncov/variants/deltavariant.html?s_cid=11512:cdc\%20delta\%20variant:sem.ga:p:RG:GM :gen:PTN:FY21.

[4] Cosdon N. Delta variant causes unprecedented COVID-19 disease in children [Internet]. Contagion Live. 2021 [cited 2021 Oct 21]. Available from: https://www.contagionlive.com/view/delta-varianticauses-unprecedented-covid-19-disease-in-children.

[5] GISAID - hCov19 Variants [Internet]. Gisaid.org. [cited 2021 Oct 21]. Available from: https://www.gisaid.org/hcov19-variants/.

[6] IANS. Delta coronavirus variant has spread to 185 countries, says WHO [Internet]. Business-standard.com. 2021 [cited 2021 Oct 24]. Available from: https://www.business-standard.com/article/currentaffairs/delta-coronavirus-variant-has-spread-to-185-countries-sayswho-121092200721_1.html.

[7] CDC. COVID Data Tracker [Internet]. Cdc.gov. 2020 [cited 2021 Oct 24]. Available from: https://covid.cdc.gov/covid-data-tracker/.

[8] CDC. What is Genomic Surveillance? [Internet]. Cdc.gov. 2021 [cited 2021 Oct 24]. Available from: https://www.cdc.gov/coronavirus/2019ncov/variants/variant-surveillance.html.
[9] Wikipedia contributors. SARS-CoV-2 Delta variant [Internet]. Wikipedia, The Free Encyclopedia. 2021 [cited 2021 Oct 24]. Available from: https://en.wikipedia.org/w/index.php?title=SARSCoV-2 Delta variant\&oldid $=1055338617$.

[10] CDC. SARS-CoV-2 Variant Classifications and Definitions [Internet]. Cdc.gov. 2021 [cited 2021 Oct 24]. Available from: https://www.cdc.gov/coronavirus/2019-ncov/variants/variantinfo.html.

[11] Gordon DE, Jang GM, Bouhaddou M, Xu J, Obernier K, White KM, et al. A SARS-CoV-2 protein interaction map reveals targets for drug repurposing. Nature. 2020; 583(7816): 459-468.

[12] Cherian S, Potdar V, Jadhav S, Yadav P, Gupta N, Das M, et al. SARSCoV-2 spike mutations, L452R, T478K, E484Q and P681R, in the second wave of COVID-19 in Maharashtra, India. Microorganisms. 2021; 9(7).

[13] Becerra A, Muñoz-Velasco I, Aguilar-Cámara A, Cottom-Salas W, Cruz-González A, Vázquez-Salazar $\mathrm{A}$, et al. Two short Low complexity regions (LCRs) are hallmark sequences of the Delta SARSCoV- 2 variant spike protein. Research Square. 2021.

[14] Chowdhury S, Bappy MH. On the delta plus variant of SARS-CoV-2. ResearchGate. [Preprint]

5] Kannan SR, Spratt AN, Cohen AR, Naqvi SH, Chand HS, Quinn TP, et al. Evolutionary analysis of the delta and delta plus variants of the SARS-CoV-2 viruses. J Autoimmun. 2021; 124(102715): 102715.

[16] CDC. Symptoms of COVID-19 [Internet]. Cdc.gov. 2021 [cited 2021 Oct 24]. Available from: https://www.cdc.gov/coronavirus/2019ncov/symptoms-testing/symptoms.html.

[17] ZOE COVID Study - Help slow the spread of COVID-19 [Internet]. Joinzoe.com. [cited 2021 Oct 24]. Available from: https://covid.joinzoe.com/us-2.

[18] FDA. Fact sheet for health care providers emergency use authorization (eua) of regen-cov [Internet]. Fda.gov. [cited 2021 Oct 24]. Available from: https://www.fda.gov/media/145611/download.

[19] FDA. Fact sheet for health care providers emergency use authorization (Eua) of bamlanivimab and etesevimab [Internet]. Fda.gov. [cited 2021 $\begin{array}{llll}\text { Oct 24]. } & \text { Available }\end{array}$ https://www.fda.gov/media/145802/download.

[20] FDA. Fact sheet for healthcare providers emergency use authorization (Eua) of sotrovimab [Internet]. Fda.gov. [cited 2021 Oct 24]. Available from: https://www.fda.gov/media/149534/download.

[21] Planas D, Veyer D, Baidaliuk A, Staropoli I, Guivel-Benhassine F, Rajah MM, et al. Reduced sensitivity of SARS-CoV-2 variant Delta to antibody neutralization. Nature. 2021; 596(7871): 276-80.

[22] Hinton DM. Convalescent Plasma EUA Letter of Authorization 06032021 [Internet]. Fda.gov. [cited 2021 Oct 24]. Available from: https://www.fda.gov/media/141477/download.

[23] UpToDate [Internet]. Uptodate.com. [cited 2021 Oct 24]. Available from: https://www.uptodate.com/contents/covid-19-management-inhospitalized-

adults? search $=$ COVID\%20treatment\&source $=$ search result\&selected Title $=1 \sim 150 \&$ usage_type $=$ default\&display_rank $=1$.

[24] Mehta P, McAuley DF, Brown M, Sanchez E, Tattersall RS, Manson JJ, et al. COVID-19: consider cytokine storm syndromes and immunosuppression. Lancet. 2020; 395(10229): 1033-1034.

[25] Lopez Bernal J, Andrews N, Gower C, Gallagher E, Simmons R, Thelwall S, et al. Effectiveness of Covid-19 vaccines against the B.1.617.2 (delta) variant. $N$ Engl J Med. 2021; 385(7): 585-594.

[26] Moline HL, Whitaker M, Deng L, Rhodes JC, Milucky J, Pham H, et $a l$. Effectiveness of COVID-19 vaccines in preventing hospitalization among adults aged $\geq 65$ years - COVID-NET, 13 states, February-April 2021. MMWR Morb Mortal Wkly Rep. 2021; 70(32): 1088-1093.

[27] Tenforde MW, Olson SM, Self WH, Talbot HK, Lindsell CJ, Steingrub JS, et al. Effectiveness of Pfizer-BioNTech and Moderna vaccines against COVID-19 among hospitalized adults aged $\geq 65$ years - United States, January-march 2021. MMWR Morb Mortal Wkly Rep. 2021; 70(18): 674-679.

[28] van Gils MJ, Lavell AHA, van der Straten K, Appelman B, Bontjer I, Poniman $\mathrm{M}$, et al. Four SARS-CoV-2 vaccines induce quantitatively different antibody responses against SARS-CoV-2 variants [Internet]. bioRxiv. [Preprint]. http://dx.doi.org/10.1101/2021.09.27.21264163.

[29] Stowe J, Andrews N, Gower C, Gallagher E, Utsi L, Simmons R, et al. Effectiveness of COVID-19 vaccines against hospital admission with the Delta (B.1.617.2) variant [Internet]. Khub.net. [Preprint] 2021. Available from: https://khub.net/web/phe-national/public-library//document_library/v2WsRK3ZlEig/view/479607266.

[30] Jagadeesh Kumar V, Sowpati DT, Munigela A, Banu S, Siva AB, Sasikala $\mathrm{M}$, et al. Clinical outcomes in vaccinated individuals 
hospitalized with Delta variant of SARS-CoV-2 [Internet]. bioRxiv. [Preprint] 2021. http://dx.doi.org/10.1101/2021.07.13.21260417.

[31] Cevik M, Grubaugh ND, Iwasaki A, Openshaw P. COVID-19 vaccines: Keeping pace with SARS-CoV-2 variants. Cell. 2021; 184(20): 5077-81.

[32] Tregoning JS, Flight KE, Higham SL, Wang Z, Pierce BF. Progress of the COVID-19 vaccine effort: viruses, vaccines and variants versus efficacy, effectiveness and escape. Nat Rev Immunol. 2021; 21(10): 626-636.

[33] Chowdhury S, Bappy MH, Chowdhury S, Chowdhury MS, Chowdhury NS. COVID-19 induced cardiovascular complications and recent therapeutic advances. EJMED. 2021;3(6): 17-22.

[34] Office of the Commissioner. FDA authorizes booster dose of PfizerBioNTech COVID-19 Vaccine for certain populations [Internet]. Fda.gov. 2021 [cited 2021 Oct 24]. Available from: https://www.fda.gov/news-events/press-announcements/fdaauthorizes-booster-dose-pfizer-biontech-covid-19-vaccine-certainpopulations.

[35] Office of the Commissioner. Coronavirus (COVID-19) update: FDA takes additional actions on the use of a booster dose for COVID-19 vaccines [Internet]. Fda.gov. 2021 [cited 2021 Oct 24]. Available from: https://www.fda.gov/news-events/press-announcements/coronaviruscovid-19-update-fda-takes-additional-actions-use-booster-dose-covid19 -vaccines.

[36] CDC. COVID-19 Vaccine Booster Shots [Internet]. Cdc.gov. 2021 [cited 2021 Oct 24]. Available from: https://www.cdc.gov/coronavirus/2019-ncov/vaccines/boostershot.html. 